\title{
SIX DOF SENSORY SYSTEM FOR THE FORCE-TORQUE CONTROL OF WALKING HUMANOID*
}

\author{
MILAN KVASNICA \\ Tomas Bata University in Zlin, Faculty of Applied Informatics \\ Nad Stránèmi 4511, CZ-76005 Zlín, Czech Republic \\ E-mail: kvasnica@fai.utb.cz.
}

\begin{abstract}
This paper focuses on current state of the art in sensory equipment for walking robotic systems and walking humanoids oriented on assistive technologies, military, security and rescue robotic systems. Described sensory systems are based on modular design that measures axial shiftings and angular displacements is a part of many sensory systems in robotics and human-machine interface applications. This is done by means of a square or annular CCD, or a set of four linear PSD elements, and four laser diode rays or planes, creating the shape of a pyramid. The positions of four light spots on the CCD or eight light spots on the PSDs are processed to produce three axial shiftings and three angular displacement values.
\end{abstract}

\section{Introduction}

The substance of six-component sensory system with the utilization for walking robotic systems is based on the shape of the 3D pyramid consisting of four edges created by light rays (respectively walls of light planes from structured light). The intersection of the laser light rays is forming the apex of the pyramid. The (square) basis of the pyramid is created by means of the 2-D Charge Coupled Device (or Position Sensitive Device, or CMOS) array and is used like a floating coordinate frame $x, y$. Intersection of four light rays with the 2-D CCD array in the basic parallel position between both two flanges results in a square shape with four light spots in the corners. Intersection of four light rays against the 2-D CCD array under acting of axial shiftings and angular displacements generally results in a trapezoidal position of the light spots. The light rays pyramid shape is coupled with the outer flange and the 2-D CCD array is coupled with the inner flange.

\footnotetext{
The financial support from the grant „Výzkumný záměr MŠMT 7088352102“ in the part „Šestisložková informace při měření statických a dynamických vlastností anizotropních materiálự" is gratefully acknowledged.
} 
The six degrees-of-Freedom (DoF) motion (three axial shiftings and three angular displacements) between two flanges is sampled by means of the unambiguous trapezoidal light spots position, see [2], [3], [4].

Simple modular construction enables low cost customization, according to the demanded properties: A -stiff module of two flanges connected by means of microelastic deformable medium; B -compliant module of two flanges connected by means of macroelastic deformable medium; $\mathrm{C}$-the module of the 2-D CCD array; D -the module of insertion flange with basic light sources configuration and focusing optics; $\mathrm{F}$-the module of the plane-focusing screen; $\mathrm{H}$ -the module of the optical member for the magnifying or reduction of the light spots configuration. The problem of the customization of six-DoF sensory systems according to the enhanced accuracy and operating frequency of the scanning of the six-DoF information is possible to improve by means of the module of insertion flange with the configuration of light sources with strip diaphragms, creating the light planes with strip light spots and by means of the module of the single or segmented linear or annular CCD or PSD elements with higher operating frequency, respectively using the module of two, parallel working, concentric CCD annulars with higher reliability.
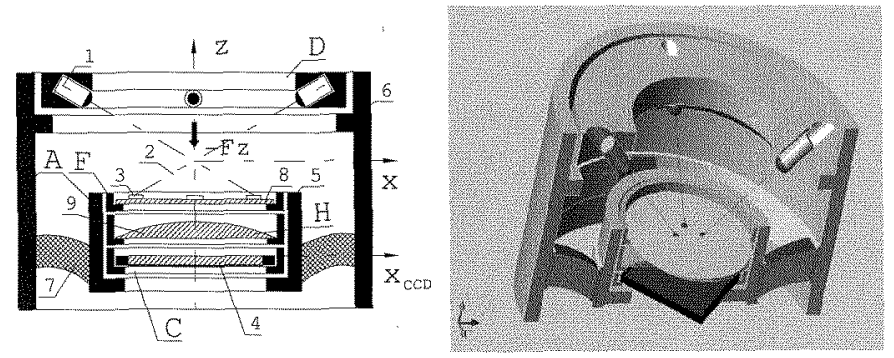

Figure 1. Six-Component Force-Torque Transducer.

The explanation of the activity of the force-torque transducer: Laser diodes 1 emit the light rays 2 creating the edges of a pyramid intersecting the plane of the 2-D CCD array, here alternatively the focusing screen 8 with light spots 3 , see Figure 1. The unique light spots configuration changes under axial shifting and angular displacements between the inner flange 5 and the outer flange 6 connected by means of elastic deformable medium 7 . An alternatively inserted optical member 9 (for the magnification of micro-movement, or the reduction of macro-movement) projects the light spots configuration from the focusing 
screen onto the 2-D CCD array 4. Four light rays simplify and enhance the accuracy of the algorithms for the evaluation of the six-DoF information.

The algorithms for the computation of three axial shiftings and three radial displacements values is based on the inverse transformation, see [11] of the final trapezoidal position of four light spots related to the original square light spots position in the plane coordinate system $x_{C C D}, y_{C C D}$ on the 2-D CCD array. This algorithm determines the relative location and orientation of a floating 2-D coordinate system against a fixed 3-D coordinate system corresponding to the apex of the pyramid shape, or contrary. The information about three axial shiftings and three angular displacements is sampled and converted according to a calibration matrix to acting forces $\mathrm{Fx}, \mathrm{Fy}, \mathrm{Fz}$ and torques $\mathrm{Mx}, \mathrm{My}, \mathrm{Mz}$, creating the vector $\mathrm{F}=[\mathrm{Fx}, \mathrm{Fy}, \mathrm{Fz}, \mathrm{Mx}, \mathrm{My}, \mathrm{Mz}]^{\mathrm{T}}$.

\section{The Design of The Calibration Equipment for the Six-Component Force-Torque Sensors}

The stability of control system of walking robots is under the influence not only static but mostly dynamic properties of acting forces $F=[F x, F y, F z$, $\mathrm{Mx}, \mathrm{My}, \mathrm{Mz}]^{\mathrm{T}}$ from the six component force-torque sensors. For the calibration of the six-component force-torque sensory systems are used two different types of calibration equipments. The first, known variant is destined for static calibration of force-torque sensors. This calibration equipment consists of six independent loading corridors for every force-torque component separately, see Figure 2. Here the acting forces Fx, Fy, Fz and torques $\mathrm{Mx}, \mathrm{My}, \mathrm{Mz}$, create the vector $\mathrm{F}=[\mathrm{Fx}, \mathrm{Fy}, \mathrm{Fz}, \mathrm{Mx}, \mathrm{My}, \mathrm{Mz}]^{\mathrm{T}}$. The vector $\mathrm{D}=\mathrm{K} . \mathrm{F}$ of axial shiftings and angular displacements on the output of the six-component sensor enables the evaluation of the vector $F=K^{(-1)} . D$, where the $K$ is the characteristic matrix and the $K^{(-1)}$ is the compensation matrix. The calibration matrix describes the properties of the elastic deformable medium in the force-torque sensor. The aim of the calibration equipment is to determine the interference of every forcetorque component to remaining components and to determine the linear part of the working characteristic.

The first variant of the calibration equipment, see [7] is destined for the static calibration which is sufficient mostly for force-torque sensory system with longer response time. Some elastic deformable medium in the force-torque sensory system are suitable only for the sampling of force-torque process with low frequency, because some materials of the elastic deformable medium interfere significant to remaining components. 


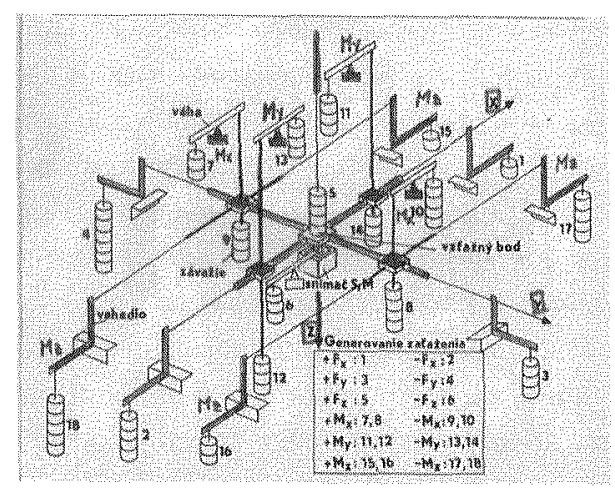

Figure 2. Calibration Equipment for Static Loading (from STU Bratislava),

The second new variant of the calibration equipment, see [10] is destined for the sampling of the six-component force-torque sensors at dynamic loading. In this calibration equipment are used the one-axis resistance strain gauges for the measurements of the force-torque components at both static and dynamic loading. The dynamic force-torque loading is acting by the use of the pressing rods moved from the mechatronic, hydraulic or pneumatic actuators against the basic points of the squirrel cage of the calibration equipment, see [5]. This enable the measurement not only the static, but as well the dynamic characteristics of the six-component force-torque sensor for more components simultaneously. The measurement frequency of dynamic calibration equipment is restricted by the maximal working frequency of strain gauges of $2 \mathrm{KHz}$, but this is sufficient for the activity of walking robots.

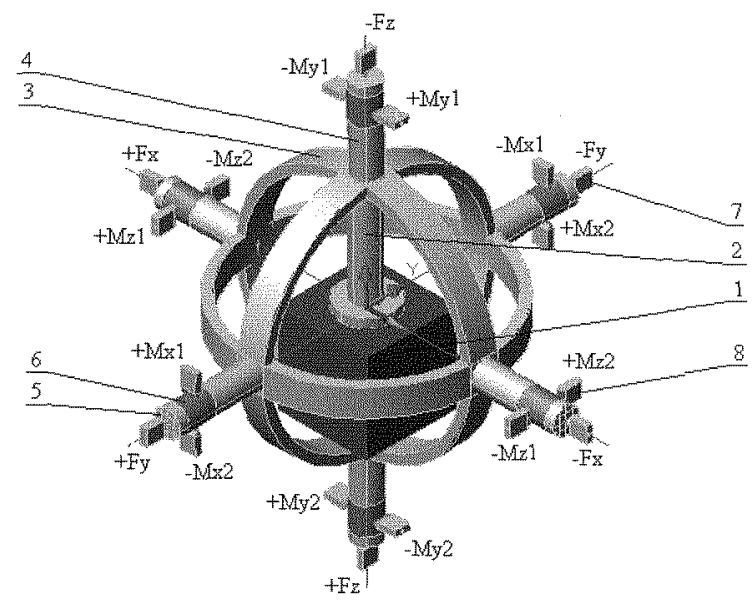

Figure 3. Calibration Equipment for Dynamic Loading (from UTB Zlin). 
In Figure 3 is depicted the design of mechanical parts of the squirrel cage between actuators and the strain gauges: 1. Six-component sensor, 2. Axial lever arm, 3. The stiff cradle, 4 . The grapple, 5 . Contact surface of the force-torque acting, 6 . Contact surface for the axial $x, y, z$ torque acting, 7 . One-axis strain gauges unit for the axial $\mathrm{x}, \mathrm{y}, \mathrm{z}$ force acting, 8 . Strain gauges units for the angular torque acting.

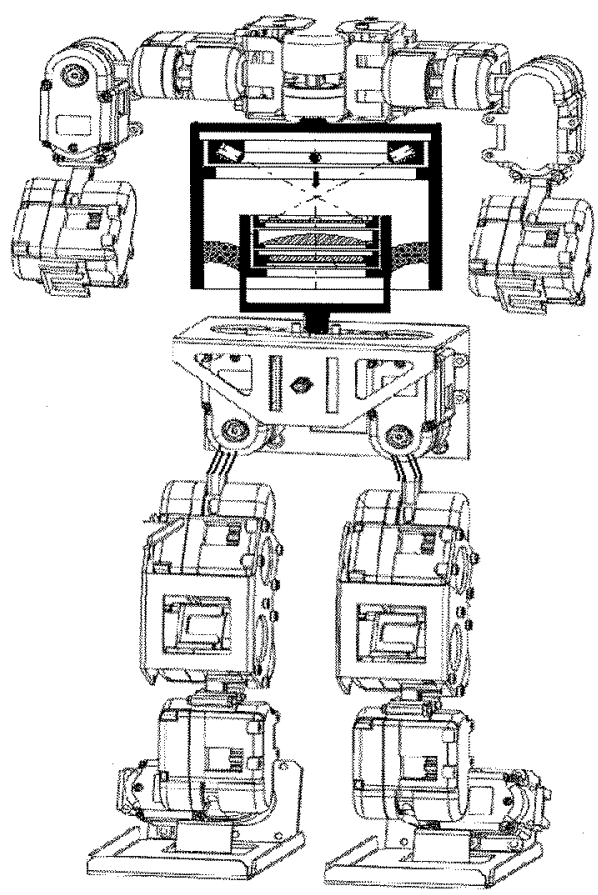

Figure 4. The Six-Component Force-Torque Transducer Inserted between the Shoulder and the Shaft of Humanoid Body.

\section{The Control of the Walking Humanoid Stability}

The six-component force-torque transducer inserted between the shoulder and the shaft of humanoid body, see [11] for the control of the stability is depicted in Figure 4 . The control of the walking humanoid's stability is parallel checked by the simple optoelectronic exteroceptive sensor for the control of a dynamic equilibrium, see [8]. This simple optoelectronic exteroceptive sensor located in 
the position of the walking robot's head is connected with the shoulder by two DoF joint servos.

\section{The Control of the Walking Leg Dynamics}

Universal, low cost, intelligent modular sensory systems enable to evaluate a humanoid's hand or leg dynamics while in motion, see [6] is depicted in Figure 5. A part of the artificial leg consists of the joint 10 connecting a shin with a foot 11 .

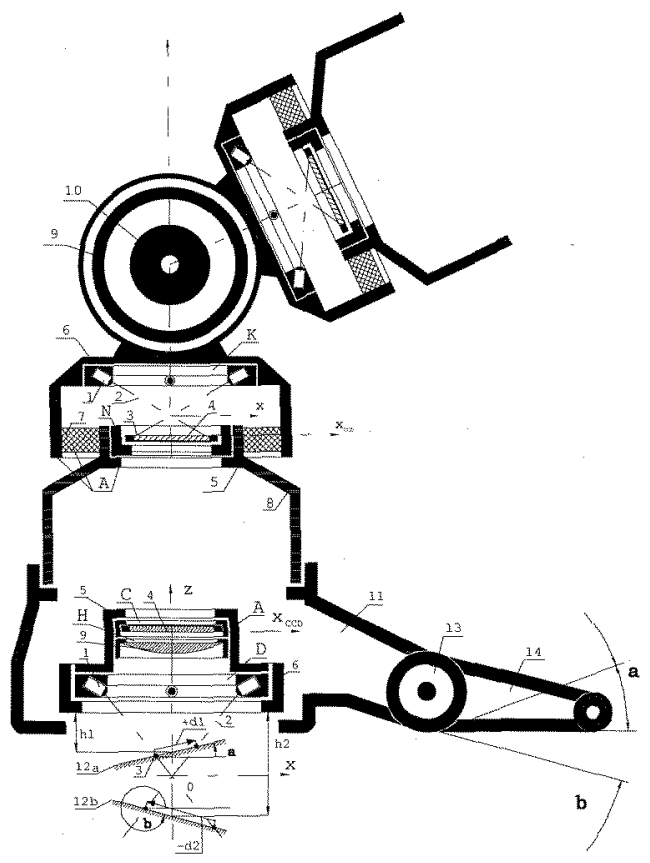

Figure 5. Six-Component Force-Torque Sensors Mounted in Humanoid's Leg and the Range-Incline Finder Built in the Heel.

The motion of the joint 11 is controlled by means of the six DoF information gained from two six-component sensors. The joint's 10 drive transmission is switched by means of the coupling muff 9 in order to control the dynamics of the motion.

The six-component information about the leg's dynamics processed from two force-torque sensors enables us to use the drive power intelligently, even to convert the damping of the joint 10 motion for energy recuperation into the battery. The joint 13 connects the foot 11 with the toes part 14 . The angular 
displacement, (here for example a, b), of the joint 13 is used for the accommodation to the ground's incline $12 \mathrm{a}, 12 \mathrm{~b}$, according to the information from the range-incline finder.

The ground's incline under the artificial leg is scanned by means of the range-incline finder mounted in a heel, see Figure 5, consisting of modules A, C, $\mathrm{D}, \mathrm{H}$. The light spots 3 from the light beams 2 on the ground $12 \mathrm{a}, 12 \mathrm{~b}$ create the configuration scanned by the 2-D CCD array. The processing of this information enables us to evaluate the incline of the ground in two mutual perpendicular planes. Real-time algorithms are suitable for the single cheap microprocessor. A microprocessor based signal processing as an indicator of the ground's incline helps the humanoid to keep the stability.

\section{Conclusion}

The modular design for six-component sensory system presented here enables easy customizing for wide variety applications in walking humanoids. Various combinations of the modular components enable tailoring of the sensory system properties including the use of the haptic interface for applications such as: detection of microelastic or macroelastic deformation, active compliant links, multi-DoF hand controllers, signature scanners, keyboards for blind people, tactile sensors, and range finders-positioners. In general, this modular design concept allows the maximization of service life because of ease of repair and the use of modular components for various types of sensors and the customization for a wide variety of design requirements. For example various levels of resolution and operating frequency, enhanced demands for safety and reliability in space robotics and medical use, and low cost design for manufacturing.

In conclusion, introduced six component sensory system enables to built walking robots appropriate for experiments in the field of assistive technologies, human-machine interface, walking platforms for military, security, antiterrorist and rescue robotics and for the swarm robotic systems.

\section{References}

1. Dobrocka E., Geometrical Principles of the Monolithic X-Ray Magnifier, Journal Appl. Cryst., 24, 212-221, (1991)

2. Hirzinger G., Dietrich J., Gombert J., Heindl J.,Landzettel K.,Schott J., The Sensory and Telerobotic Aspects of Space Robot Technology Experiment ROTEX, Proceedings of the International Symposium on Artificial Intelligence, Robotics and Automation in Space, Toulouse, Labege, France, (1992). 
3. Kvasnica M., Intelligent Sensors for the Control of Autonomous Vehicles, Proceedings of the 6th International Conference and Exposition on Engineering, Construction and Operation in Space and on Robotics for Challenging Environments - Space and Robotics 98, Albuquerque, New Mexico, USA, (1998).

4. Kvasnica M., Improvement of Positioning Accuracy in Multi-Pod Parallel Structures", ASCE Multi-Conference on Engineering, Construction, Operations, and Business in Space and on Robotics for Challenging Situations and Environment "Space and Robotics 2002, Albuquerque, New Mexico, USA, (2002).

5. Palka M., The Design of the Calibration of the Six-Component ForceTorque Sensor, Diploma Thesis, Tomas Bata University in Zlin, Czech Republic, (2007).

6. Kvasnica M., Modular Force-Torque Transducers for Rehabilitation Robotics, Proceedings of the 6th International Conference on Rehabilitation Robotics ICORR'1999, Stanford University, (1999).

7. Sásik J., Multi-component Force-Torque Sensors Calibration Methods for Robotics Application, Strojnícky časopis, 38, No.6, Bratislava, Slovakia, (1987).

8. Kral E., Simple optoelectronic exteroceptive sensor for the control of a dynamic equilibrium of a walking robot. Proceedings of the 11th International Conference on Climbing and Walking Robots and the Support Technologies for Mobile Machines, Coimbra, Portugal, (2008).

9. Kvasnica M., Algorithmic Approach for the Sampling of Six Degrees of Freedom Information Using Floating 2-D Coordinate Frame. Proceedings of the International Workshop on Robotics and Mathematics ROBOMAT 2007, Coimbra, Portugal, (2007).

10. Kvasnica M., The Design of the Calibration Equipment for the Six Component Force-Torque Sensor. Proceedings of the International Congress Mechatronics and Robotícs MiR 2007, Izdatělstvo Poligrafičeskij Komplex Lenexpo, Saint Petersburg, Russia, (2007).

11. http://www.megarobot.net 